\title{
Florística e fitossociologia em um trecho de restinga no Litoral Norte do Estado da Bahia
}

\author{
Christiano Marcelino Menezes ${ }^{1}$ \\ Flávia Delgado Santana ${ }^{2}$ \\ Vinicius Sancho A. Silva ${ }^{3}$ \\ Vanessa Iris Silva da Silva ${ }^{1 *}$ \\ Dorothy Sue Dunn Araújo 4

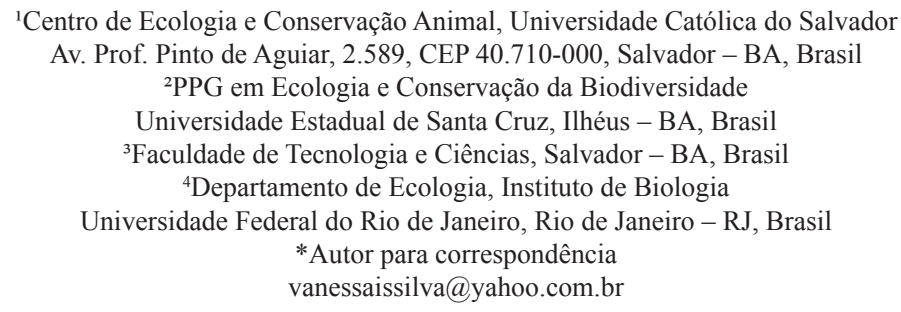

Submetido em 03/03/2011

Aceito para publicação em 05/11/2011

\section{Resumo}

As restingas são ambientes geologicamente recentes e as espécies que as habitam são típicas de ecossistemas adjacentes como as Florestas Ombrófilas, as Matas de Tabuleiro e a Caatinga, sendo este, o aspecto mais importante na sua composição de espécies, que é composta por diversas formas biológicas, tais como árvores, arbustos, herbáceas eretas e reptantes. O estudo foi realizado no Município de Mata de São João na vila de Praia do Forte, localizada ao longo do Litoral Norte do Estado da Bahia. Com o objetivo de avaliar se há existência de diferentes comunidades vegetais em um trecho de restinga, foram locadas quatro transecções de $200 \mathrm{~m}$ com cinco unidades amostrais de $100 \mathrm{~m}^{2}$ cada. Ao final do estudo foram amostrados 1.640 indivíduos distribuídos em 68 espécies. Os dados florísticos e fitossociológicos associados à análise de Cluster apontam a existência de duas comunidades vegetais distintas, sendo elas uma Mata de Restinga (Transecções 3 e 4) e uma Restinga em Moitas (Transecções 1 e 2). Uma elevada diversidade de espécies foi detectada nas transecções 1 e 2 (H' $=3,882)$ e 3 e $4\left(H^{\prime}=3,835\right)$, além de uma diferença significativa na composição das espécies entre elas ( $p<$ 0,001). As famílias mais representativas em número de espécies foram: Myrtaceae, Rubiaceae e Malpighiaceae.

Palavras-chave: Fitossociologia, Florística, Restinga

\section{Abstract}

Flora and phytosociology of an area of Restinga along the northern Coast of Bahia, Brazil. Areas of restinga are geologically recent environments. The species that inhabit this type of ecosystem are typical of adjacent areas, such as rain forest, tabuleiro forest and caatinga, and form a diverse community of trees, shrubs and erect and prostrate herbs. The goal of this work was to evaluate the vegetation in an area of restinga located 
along Praia do Forte, in the state of Bahia, Brazil. Four 200m transects where made within five plots of $100 \mathrm{~m}^{2}$ each, and 1,640 individuals within 68 species were collected. High species diversity was found in transects 1 and $2\left(\mathrm{H}^{\prime}=3.882\right)$ and 3 and $4\left(\mathrm{H}^{\prime}=3.835\right)$. The most representative families were Myrtaceae, Rubiaceae and Malpighiaceae. Based on the species composition, a significant difference $(\mathrm{p}<0.001)$ was found between the sampled areas, and the findings show that there are two distinct plant communities in this region, Mata de Restinga and Restinga em Moitas.

Key words: Flora, Phytosociology, Restinga

\section{Introdução}

As restingas são ambientes geologicamente recentes e as espécies que as habitam são típicas de ecossistemas adjacentes como a Mata Atlântica, a Mata de Tabuleiro e a Caatinga (FREIRE, 1990). Segundo Martins et al. (2008), as restingas são complexas, frágeis e altamente afetadas pelo desmatamento, além de dependerem da dinâmica hídrica das áreas onde ocorrem. Os mesmos autores afirmam ainda que o desmatamento e a consequente retirada de camadas superficiais do solo interferem no processo sucessional das restingas, podendo ser reversível somente a longos prazos. Segundo Menezes (2007), as restingas localizamse em áreas de sedimentação quaternária, enquanto que Rizzini (1997) afirma que as restingas são formações vegetais colonizadoras de areias holocênicas. O solo se torna um fator importante para a composição da vegetação (SILVA, 1998), que é composta por diversas formas biológicas vegetais, tais como árvores, arbustos, herbáceas eretas e reptantes (PEREIRA et al., 1992).

O Litoral Norte da Bahia se estende por aproximadamente $200 \mathrm{~km}$, estando limitado ao sul pela cidade do Salvador e ao Norte pelo Rio Real na divisa com o Estado de Sergipe. Em 21 de fevereiro de 1995, através da resolução $\mathrm{n}^{\circ} 1.040$ foi aprovada a criação da Área de Proteção Ambiental - Litoral Norte. A APA Litoral Norte - oficializada pelo Decreto Estadual de $n^{\circ} 1.046$, de 17/03/1992, abrange os municípios de Jandaíra, Esplanada, Conde, Entre Rios e Mata de São João. A APA apresenta 142.000ha, sendo a segunda maior da Bahia. Sua criação foi fundamentada na necessidade de conservar e preservar os remanescentes da Mata Atlântica e ecossistemas associados como os manguezais, áreas estuarinas, restingas, dunas e lagoas. Além disso, com a implantação da Linha Verde (BA - 099), o processo de ocupação tornou-se intenso juntamente com o turismo, trazendo assim, a necessidade de um plano de manejo para resguardar os recursos ambientais, ordenando o uso e ocupação do solo.

Poucos são os trabalhos publicados sobre a vegetação de restinga do Litoral Norte do Estado da Bahia, com exceção das publicações de Britto et al. (1993), realizado nas dunas e lagoas do Abaeté, no município de Salvador, o de Pinto et al. (1984), que se destaca por ter sido o primeiro estudo publicado que encerra descrição de aspectos fitofisionômicos para o Litoral Norte do Estado, um check-list das espécies vegetais ocorrentes no Litoral Norte da Bahia, Costa dos Coqueiros e Salvador, publicado pelo IBGE (2004) e mais recentemente os estudos de Queiroz (2001), Menezes (2007) e Menezes et al. (2009).

Diante desses aspectos é que se objetivou avaliar se há existência de diferentes comunidades vegetais em um trecho de restinga na Vila de Praia o Forte, município de Mata de São João, Bahia.

\section{Material e Métodos}

\section{Área de estudo}

Localização - As áreas de amostragem selecionadas encontram-se dentro dos limites do Complexo Hoteleiro Iberostar, localizado na APA - Área de Proteção Ambiental do Litoral Norte da Bahia, situado na Vila de Praia do Forte, distando cerca de $60 \mathrm{~km}$ da cidade do Salvador, podendo ser acessado pela BA-099 (Figura 1).

Geomorfologia e solos - Os substratos são classificados pelos tipos podzólicos vermelho amarelo e areias quartzozas. Estes tipos de substratos são decorrentes da Formação Barreiras do Terciário. Seus sedimentos são formados por conglomerados 
poligênicos, consolidados e depositados de forma variada, o que lhes confere uma textura fina e com boa permeabilidade e porosidade (FUNATURA, 1987).

FIGURA 1: Croqui de localização da área de estudo, Praia do Forte, Município de Mata de São João-BA.

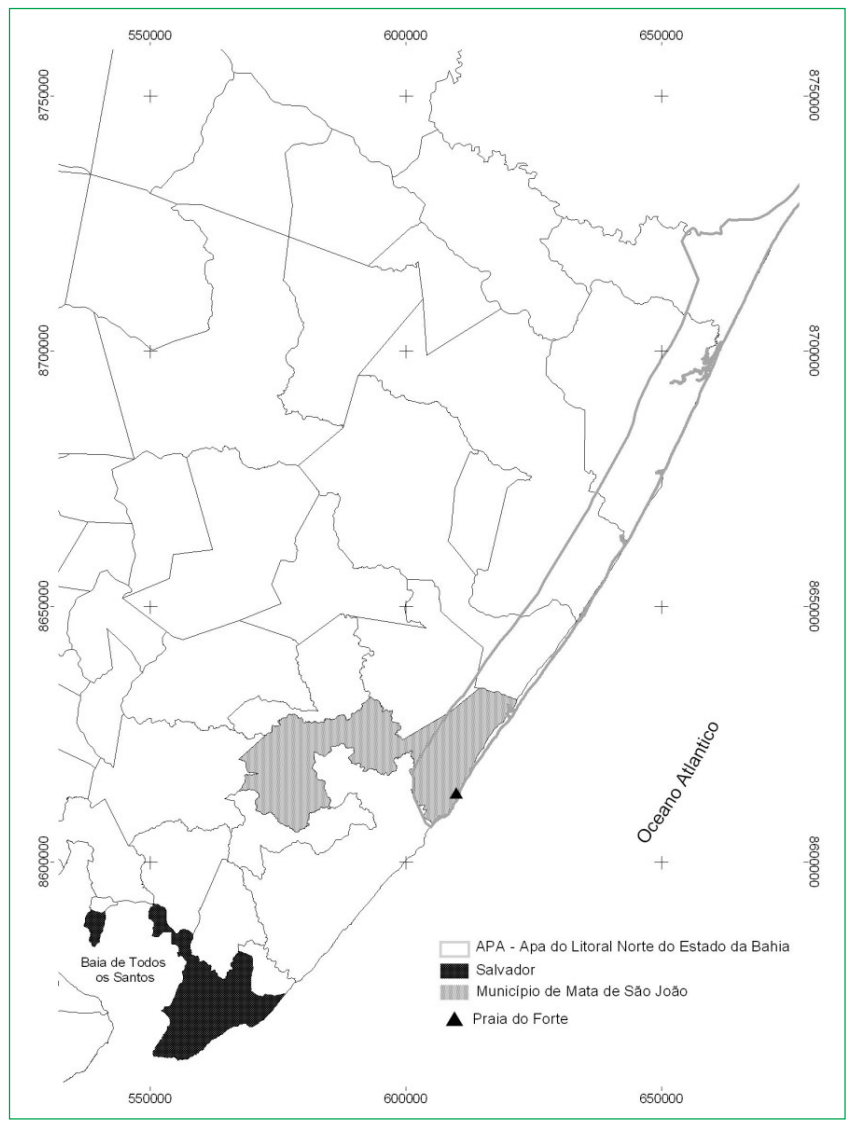

Clima - Pode ser citado como tropical, sempre úmido, com temperatura média anual de $25^{\circ} \mathrm{C}$. Nos meses mais frios, julho e agosto, a temperatura fica abaixo dos $18^{\circ} \mathrm{C}$, sendo a pluviosidade em torno de 2.000mm anuais (FUNATURA, 1987). De acordo com a classificação de Köppen na região, Funatura, 1987 cita que, dentro da localidade em estudo, não possui estação seca definida e a concentração das chuvas ocorre entre os meses de março a agosto.

Vegetação - A vegetação da área de estudo está inserida no Quaternário Costeiro do Litoral Norte do Estado em domínio do ecossistema de Restinga. Dois estratos vegetacionais são reconhecidos: um arbustivo-arbóreo e um arbóreo (MENEZES; FARIA, 2004), cujos seus limites e suas espécies características são desconhecidas. Segundo esses autores, a vegetação tende a aumentar em sentido oposto ao da linha de praia.

\section{Delineamento amostral}

Foi utilizado o método de parcelas (unidades amostrais) múltiplas (MULLER-DOMBOIS; ELLENBERG, 1974), distribuídas ao longo de quatro transecções lineares com $200 \mathrm{~m}$ de extensão, sendo que cada uma apresentava cinco unidades amostrais de $10 \times 10 \mathrm{~m}$ e eqüidistantes entre si por $50 \mathrm{~m}$. As transecções 1 e 2 estão distanciadas a aproximadamente $360 \mathrm{~m}$ da linha de praia, enquanto que a 3 e 4 com a aproximadamente $800 \mathrm{~m}$ (Figuras 2 e 3 ).

FIGURA 2: Croqui de localização das transecções utilizadas para a distribuição das unidades amostrais.

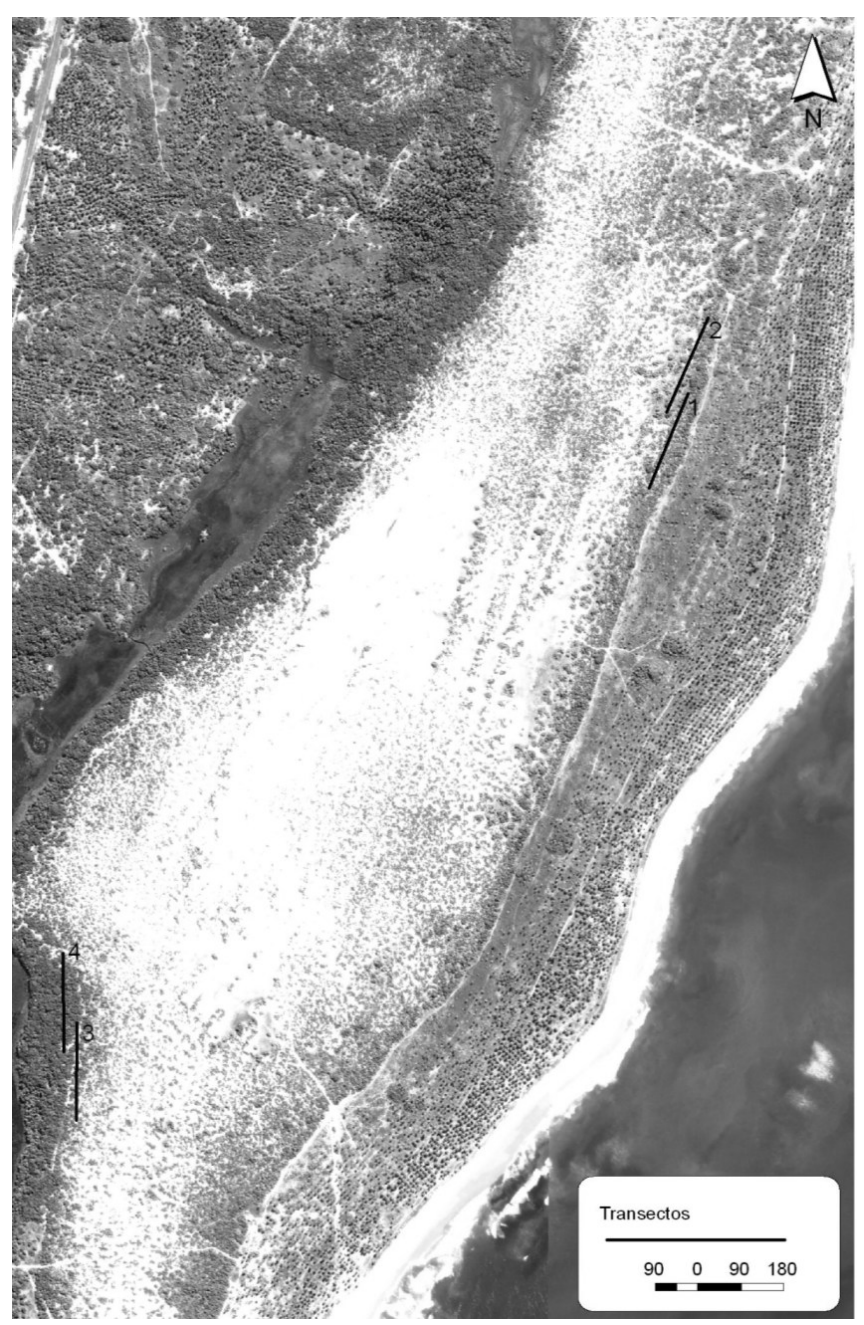


FIGURA 3: Localização das transecções onde foram dispostas as unidades amostrais.

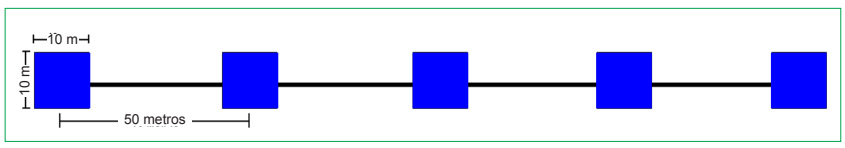

\section{Coleta de dados}

Em cada unidade amostral, foram analisados todos os indivíduos lenhosos com CAS (Circunferência a Altura do Solo) de $\geq 5 \mathrm{~cm}$, que estivessem totalmente ou parcialmente inseridos nas unidades amostrais, sendo a altura estimada visualmente. Todos os dados foram registrados em cadernetas de campo.

\section{Identificação botânica}

Quando necessário, foram escolhidos exemplares férteis e coletados para identificação. Estes foram prensados e herborizados segundo as técnicas usuais (BRIDSON; FORMAN, 1992), triados no laboratório do Centro de Ecologia Conservação Animal - ECOA/UCSal, e encaminhados ao acervo do Herbário RADAMBRASIL (HRB) no Jardim Botânico do Salvador (JBSSA). Para os indivíduos que se encontravam estéreis (sem flores e frutos), a identificação botânica se deu em campo, já que os sistemas reprodutivos são utilizados na identificação das plantas superiores. Neste sentido, foi dada ênfase a bibliografia específica (BARROSO et al., 1978; 1984; 1986; LORENZI, 1992a; 1992b; LORENZI et al., 1996; SOUZA; LORENZI, 2005). A revisão nomenclatural foi feita através do site International Plant Names Index (http://www.ipni.org/ipni/plantnamesearchpage. do) e Brummitt e Powell (1992), enquanto que para a classificação dos taxa foi utilizado o sistema APG II (2003).

\section{Fitossociologia}

Os parâmetros fitossociológicos calculados foram: Densidades Absoluta e Relativa (DA e DR), Frequências Absoluta e Relativa (FA e FR) e o Índice de Valor de Importância (IVI). Os valores de densidade relativa e frequência relativa foram utilizados para determinar o VI, já que é o investigador quem determina qual destes é o mais importante para alcançar os objetivos da pesquisa (MUELLER-DOMBOIS; ELLENBERG, 1974; FELFILI; REZENDE, 2003).

\section{Análise de cluster}

Para análise e comparação da composição das espécies entre as áreas amostradas, foi utilizado o método do Procedimento de Permutação de Reposta Múltipla (MRPP), através do uso do programa PC-ORD ${ }^{\circ}$, o qual permitiu uma análise de Cluster. A diversidade de espécies foi calculada segundo Índice de Shannon (H') (KREBS, 1999). Para análise da variância foi utilizado o método do Procedimento de Permutação de Reposta Múltipla (MRPP), através do uso do Programa PC-ORD ${ }^{\circ}$.

\section{Resultados e Discussão}

Foram amostrados no total 1.640 indivíduos, distribuídos em 68 espécies. As famílias mais representativas em número de espécies foram Myrtaceae, Rubiaceae e Malpighiaceae. Para as Matas Arenosas do Parque Estadual de Itapuã, no Rio Grande do Sul, a família Myrtaceae também foi indicada como a de maior riqueza de espécies (SCHERER \& MARASCHINSILVA, BAPTISTA, 2005), o mesmo acontecendo para as restingas do litoral de Pernambuco, onde as famílias Myrtaceae e Rubiaceae, assim como no presente estudo, foram as mais representativas em número de espécies (SACRAMENTO et al., 2007). Em um trecho de vegetação de restinga do Parque Estadual do Rio da Onça em Matinhos no Estado do Paraná, a família Myrtaceae se apresentou entre as famílias mais ricas em espécies (SONEHARA, 2005). Nas transecções 1 e 2 foram identificadas 36 espécies distribuídas em 677 indivíduos, enquanto que nas 3 e 4 foram amostradas 32 espécies em um total de 963 indivíduos. Uma elevada diversidade de espécies foi detectada nas transecções $1 \mathrm{e}$ $2\left(\mathrm{H}^{\prime}=3,882\right) 3$ e $4\left(\mathrm{H}^{\prime}=3,835\right)$. Ocorreram 16 espécies exclusivas na transecção 3 e 4, a exemplo Tabebuia elliptica (DC.) Sandwith, Syagrus schizophylla (Mart.) e Lippia iodophylla Schauer e 15 espécies exclusivas nas transecções 1 e 2, como por exemplo, Maprounea guianensis Aubl. e Myrsine sp.. Uma diferença extremamente significativa na composição das espécies entre as áreas amostradas foi detectada $(\mathrm{p}=0,00151914)$. 
As espécies de maior Valor de Importância (VI) nas transecções 01 e 02 foram: Croton selowii $(32,85)$, Coccoloba alnifolia (19,55), Manilkara salzmanii (17,39), Alibertia sp., (15,32), Byrsonima blanchetiana $(13,25)$, Myrciaria floribunda (11,58), Myrcia guianensis $(9,32)$, Maytenus sp. (7,51), Myrcine sp. (5,73), Guettarda platypoda (5,59), Clusia hilariana (5,29), Esenbeckia grandifolia $(4,07)$ e Coccoloba leavis $(3,77)$, e juntas representaram cerca de $70 \%$ do Valor de Importância Total (VI) nestas unidades amostrais (Tabela 1).
Dentre as espécies de maior valor de importância (VI) nas transecções 3 e 4 destacam-se Esenbeckia grandiflora $(24,65)$, Myrcia guianensis (21,93), Myrciaria floribunda (16,35), Stigmaphyllon paralias (15,86), Guettarda platypoda (14,04), Croton sellowii (12,97), Alibertia sp. $(12,89)$, Manilkara salzmanii $(12,06)$ e Coccoloba sp. $(10,35)$ (Tabela 2), e somadas representaram cerca das $70 \%$ das espécies dominantes. Em uma área localizada a $15 \mathrm{~km}$ da área do presente estudo, Menezes (2007) também reconheceu Coccoloba alnifolia, Manilkara

TABELA 1: Dados fitossociológicos das espécies amostradas nas transecções 1 e 2 $(\mathrm{DA}=$ Densidade Absoluta, $\mathrm{DR}=$ Densidade Relativa, $\mathrm{FA}=$ Frequência Absoluta, FR = Frequência Relativa, VI = Valor de Importância).

\begin{tabular}{|c|c|c|c|c|c|}
\hline Espécies & $\overline{\mathbf{D A}}$ & $\overline{D R}$ & $\overline{\text { FA }}$ & FR & VI \\
\hline Croton selowii & 1760 & $26,00 \%$ & 1,00 & $6,85 \%$ & 32,85 \\
\hline Coccoloba alnifolia & 860 & $12,70 \%$ & 1,00 & $6,85 \%$ & 19,55 \\
\hline Manilkara salzmanii & 760 & $11,23 \%$ & 0,90 & $6,16 \%$ & 17,39 \\
\hline Alibertia sp. & 620 & $9,16 \%$ & 0,90 & $6,16 \%$ & 15,32 \\
\hline Byrsonima blanchetiana & 480 & $7,09 \%$ & 0,90 & $6,16 \%$ & 13,25 \\
\hline Myrciaria floribunda & 320 & $4,73 \%$ & 1,00 & $6,85 \%$ & 11,58 \\
\hline Myrcia guianensis & 260 & $3,84 \%$ & 0,80 & $5,48 \%$ & 9,32 \\
\hline Maytenus sp. & 230 & $3,40 \%$ & 0,60 & $4,11 \%$ & 7,51 \\
\hline Myrcine sp. & 110 & $1,62 \%$ & 0,60 & $4,11 \%$ & 5,73 \\
\hline Guettarda platypoda & 100 & $1,48 \%$ & 0,60 & $4,11 \%$ & 5,59 \\
\hline Clusia hilariana & 80 & $1,18 \%$ & 0,60 & $4,11 \%$ & 5,29 \\
\hline Esenbeckia grandifolia & 90 & $1,33 \%$ & 0,40 & $2,74 \%$ & 4,07 \\
\hline Coccoloba leavis & 70 & $1,03 \%$ & 0,40 & $2,74 \%$ & 3,77 \\
\hline Capparis flexuosa & 70 & $1,03 \%$ & 0,40 & $2,74 \%$ & 3,77 \\
\hline Ouratea suaveolans & 110 & $1,62 \%$ & 0,30 & $2,05 \%$ & 3,68 \\
\hline Maprounea guianensis & 100 & $1,48 \%$ & 0,30 & $2,05 \%$ & 3,53 \\
\hline Erythroxylon passerinum & 80 & $1,18 \%$ & 0,30 & $2,05 \%$ & 3,24 \\
\hline Guapira pernambucensis & 70 & $1,03 \%$ & 0,30 & $2,05 \%$ & 3,09 \\
\hline Indet 04 & 60 & $0,89 \%$ & 0,30 & $2,05 \%$ & 2,94 \\
\hline Indet 03 & 60 & $0,89 \%$ & 0,30 & $2,05 \%$ & 2,94 \\
\hline Diospyros sp. & 60 & $0,89 \%$ & 0,30 & $2,05 \%$ & 2,94 \\
\hline Chioccoca alba & 50 & $0,74 \%$ & 0,30 & $2,05 \%$ & 2,79 \\
\hline Indet 01 & 40 & $0,59 \%$ & 0,30 & $2,05 \%$ & 2,65 \\
\hline Byrsonimia sp. & 40 & $0,59 \%$ & 0,30 & $2,05 \%$ & 2,65 \\
\hline Myrcia sp. & 30 & $0,44 \%$ & 0,20 & $1,37 \%$ & 1,81 \\
\hline Indet 02 & 30 & $0,44 \%$ & 0,20 & $1,37 \%$ & 1,81 \\
\hline Inga afinnis & 20 & $0,30 \%$ & 0,20 & $1,37 \%$ & 1,67 \\
\hline Myrcia rostrata & 50 & $0,74 \%$ & 0,10 & $0,68 \%$ & 1,42 \\
\hline Waltheria cinerascens & 40 & $0,59 \%$ & 0,10 & $0,68 \%$ & 1,28 \\
\hline Anacardium occidentale & 40 & $0,59 \%$ & 0,10 & $0,68 \%$ & 1,28 \\
\hline Xylopia leavigata & 30 & $0,44 \%$ & 0,10 & $0,68 \%$ & 1,13 \\
\hline Vernonia sp. & 10 & $0,15 \%$ & 0,10 & $0,68 \%$ & 0,83 \\
\hline Stigmaphyllon paralias & 10 & $0,15 \%$ & 0,10 & $0,68 \%$ & 0,83 \\
\hline Rutaceae & 10 & $0,15 \%$ & 0,10 & $0,68 \%$ & 0,83 \\
\hline Emottum affine & 10 & $0,15 \%$ & 0,10 & $0,68 \%$ & 0,83 \\
\hline Davilla sp. & 10 & $0,15 \%$ & 0,10 & $0,68 \%$ & 0,83 \\
\hline Total & 6770 & $100,00 \%$ & 14,60 & $100,00 \%$ & 200 \\
\hline
\end{tabular}


salzmanii, Myrcia guianensis, Byrsonima blanchetiana e Alibertia sp. como dominantes em uma Restinga em Moitas, assim como Guettarda platypoda e Manilkara salzmanii para as fisionomias florestais estudadas.

Myrcia guianensis e Myrciaria floribunda na transecção 1, apresentaram 4,73\% e 3,84\% da Densidade Relativa Total, respectivamente, enquanto que nas transecções 3 e 4 essas mesmas espécies apresentaram-se entre as mais abundantes, e com $15,16 \%$ e $8,83 \%$ da densidade relativa total, as mesmas espécies com valores bem diferenciados dentro das unidades amostrais citadas.

Vale ressaltar que Coccoloba alnifolia, espécie dominante nas transecções 1 e 2, não ocorreu nas transcções 3 e 4 e Esenbeckia grandiflora, dominante nas transecções 3 e 4, nas transecções 1 e 2 ocorreu apenas na décima segunda posição quando considerado o valor de importância. Vale salientar, que a dominância da espécie Croton selowii, tipicamente ruderal nas áreas amostradas, denota degradação na vegetação local.

TABELA 2: Dados fitossociológicos das espécies amostradas nas transecções 3 e 4 (DA $=$ Densidade Absoluta, DR $=$ Densidade Relativa, FA = Frequência Absoluta, $\mathrm{FR}=$ Frequência Relativa, VI = Valor de Importância).

\begin{tabular}{|c|c|c|c|c|c|}
\hline$\overline{\text { Espécies }}$ & $\overline{\mathbf{D A}}$ & $\overline{\text { DR }}$ & $\overline{\text { FA }}$ & $\overline{\text { FR }}$ & VI \\
\hline Esenbeckia grandiflora & 1650 & $17,13 \%$ & 1,00 & $7,52 \%$ & 24,65 \\
\hline Myrcia guianensis & 1460 & $15,16 \%$ & 0,90 & $6,77 \%$ & 21,93 \\
\hline Myrciaria floribunda & 850 & $8,83 \%$ & 1,00 & $7,52 \%$ & 16,35 \\
\hline Stigmaphyllon paralias & 1310 & $13,60 \%$ & 0,30 & $2,26 \%$ & 15,86 \\
\hline Guettarda platypoda & 700 & $7,27 \%$ & 0,90 & $6,77 \%$ & 14,04 \\
\hline Croton sellowii & 670 & $6,96 \%$ & 0,80 & $6,02 \%$ & 12,97 \\
\hline Alibertia sp. & 590 & $6,13 \%$ & 0,90 & $6,77 \%$ & 12,89 \\
\hline Manilkara salzmanii & 510 & $5,30 \%$ & 0,90 & $6,77 \%$ & 12,06 \\
\hline Coccoloba sp. & 490 & $5,09 \%$ & 0,70 & $5,26 \%$ & 10,35 \\
\hline Tabebuia eliptica & 270 & $2,80 \%$ & 0,80 & $6,02 \%$ & 8,82 \\
\hline Byrsonima blanchetiana & 290 & $3,01 \%$ & 0,70 & $5,26 \%$ & 8,27 \\
\hline Guapira pernambucensis & 90 & $0,93 \%$ & 0,60 & $4,51 \%$ & 5,45 \\
\hline Lippia iodophylla & 80 & $0,83 \%$ & 0,40 & $3,01 \%$ & 3,84 \\
\hline Coccoloba alnifolia & 50 & $0,52 \%$ & 0,40 & $3,01 \%$ & 3,53 \\
\hline Indet 01 & 50 & $0,52 \%$ & 0,40 & $3,01 \%$ & 3,53 \\
\hline Syagrus shizophylla & 150 & $1,56 \%$ & 0,20 & $1,50 \%$ & 3,06 \\
\hline Maytenus sp. & 70 & $0,73 \%$ & 0,30 & $2,26 \%$ & 2,98 \\
\hline Chioccoca alba & 30 & $0,31 \%$ & 0,30 & $2,26 \%$ & 2,57 \\
\hline Vernonia sp. & 60 & $0,62 \%$ & 0,20 & $1,50 \%$ & 2,13 \\
\hline Myrtaceae sp.02 & 30 & $0,31 \%$ & 0,20 & $1,50 \%$ & 1,82 \\
\hline Xylopia sp. & 20 & $0,21 \%$ & 0,20 & $1,50 \%$ & 1,71 \\
\hline Ocotea sp. & 50 & $0,52 \%$ & 0,10 & $0,75 \%$ & 1,27 \\
\hline Calycolpus legrandii & 40 & $0,42 \%$ & 0,10 & $0,75 \%$ & 1,17 \\
\hline Myrcia sp. & 20 & $0,21 \%$ & 0,10 & $0,75 \%$ & 0,96 \\
\hline Ternstroemia brasiliensis & 20 & $0,21 \%$ & 0,10 & $0,75 \%$ & 0,96 \\
\hline Araliaceae & 10 & $0,10 \%$ & 0,10 & $0,75 \%$ & 0,86 \\
\hline Coccoloba molis & 10 & $0,10 \%$ & 0,10 & $0,75 \%$ & 0,86 \\
\hline Erythroxylon passerinum & 10 & $0,10 \%$ & 0,10 & $0,75 \%$ & 0,86 \\
\hline Inga sp. & 10 & $0,10 \%$ & 0,10 & $0,75 \%$ & 0,86 \\
\hline Myrtaceae sp.01 & 10 & $0,10 \%$ & 0,10 & $0,75 \%$ & 0,86 \\
\hline Ouratea suaveolans & 10 & $0,10 \%$ & 0,10 & $0,75 \%$ & 0,86 \\
\hline Schefflera sp. & 10 & $0,10 \%$ & 0,10 & $0,75 \%$ & 0,86 \\
\hline Waltheria cinerascens & 10 & $0,10 \%$ & 0,10 & $0,75 \%$ & 0,86 \\
\hline Total & 9630 & $100,00 \%$ & 13,30 & $100,00 \%$ & 200 \\
\hline
\end{tabular}


Considerando-se a heterogeneidade observada $(\mathrm{p}=0,00151914)$ e os resultados da análise de Cluster, percebe-se a existência de dois grandes grupos vegetais (Figura 4). A vegetação amostrada nas transecções 1 e 2 correspondem a uma Restinga em Moitas, enquanto que nas transecções 3 e 4 a uma Mata de Restinga, já que espécies arbóreas típicas desta Mata ocorreram exclusivamente ou foram muito abundantes nessas transecções como: Ocotea notata, Calycolppus legrandii, Inga affine, Myrciaria floribunda e Erythroxylon passerinum. A ocorrência de Clusia hilariana em posição central e destacada nas moitas das transecções 1 e 2, assemelha-se a dados obtidos para as restingas do Rio de Janeiro (HENRIQUES et al., 1986; MONTEZUMA, 1997; ZALUAR, 1997; ASSUMPÇÃO; NASCIMENTO, 2000; ZALUAR; SCARANO, 2000), que sugere um comportamento focal desta espécie.

A heterogeneidade da vegetação de restinga apontada por outros autores para diferentes trechos da costa brasileira também foi evidenciada na área estudada. Mesmo dentro de um pequeno trecho da Planície Quaternária local, foi possível o reconhecimento de duas comunidades vegetais distintas. Tal fato demonstra a complexidade ecológica a qual as comunidades vegetais de restinga estão inseridas, principalmente no que tange a sua relação com os diferentes tipos geomorfológicos existentes no litoral brasileiro.

\section{Referências}

APG II - ANGIOSPERM PHYLOGENY GROUP. An update of the angiosperm phylogeny group classification of the orders and families of flowering plants: APGII. Botanical Journal of the Linnean Society, London, v. 141, p. 399-436, 2003.

ASSUMPÇÃO, J.; NASCIMENTO, M. T. Estrutura e composição florística de quatro formações vegetais de restinga no complexo Lagunar Grussaí/Iquipari, São João da Barra, RJ, Brasil. Acta Botanica Brasilica, Feira de Santana, v. 14, n. 3, p. 301-315, 2000.

BARROSO, G. M.; PEIXOTO, A. L.; ICHASO, C. L. F.; GUIMARÃES, E. F.; COSTA, C. G. Sistemática de angiospermas do Brasil. Vol. 1. São Paulo: EDUSP. 1978. 255 p.

BARROSO, G. M.; PEIXOTO, A. L.; ICHASO, C. L. F.; GUIMARÃES, E. F.; COSTA, C. G. Sistemática de angiospermas do Brasil. v. 2. São Paulo: Livros Técnicos e Científicos/EDUSP. 1984. 377 p.

BARROSO, G. M.; PEIXOTO, A. L.; ICHASO, C. L. F.; GUIMARÃES, E. F.; COSTA, C. G. Sistemática de angiospermas do Brasil. v. 3. São Paulo: Livros Técnicos e Científicos/EDUSP. 1986. $326 \mathrm{p}$.

BRIDSON, D.; FORMAN, L. The herbarium handbook. London: Royal Botanic Gardens Kew. 1992. 303 p.

BRITTO, I. C.; QUEIROZ, L. P.; GUEDES, M. L. S.; OLIVEIRA, N. C.; SILVA, L. B. Flora fanerogâmica das dunas e lagoas de Abaeté, Salvador, Bahia. Sitientibus, Feira de Santana, v. 11, p. 31-46, 1993. BRUMMITT, R. K.; POWELL, C. E. Authors of plant names. London: Royal Botanic Gardens Kew. 1992. 732 p.

FELFILI, J. M.; REZENDE, R. P. Conceitos e métodos em fitossociologia. Brasília: Universidade de Brasília, Departamento de Engenharia Florestal. 2003. 68 p.

FREIRE, M. S. B. Levantamento florístico do Parque Estadual das Dunas de Natal. Acta Botanica Brasilica, Feira de Santana, v. 4, p. 41-59, 1990.

FIGURA 4: Dendrograma da análise de cluster (PC-ORDC).

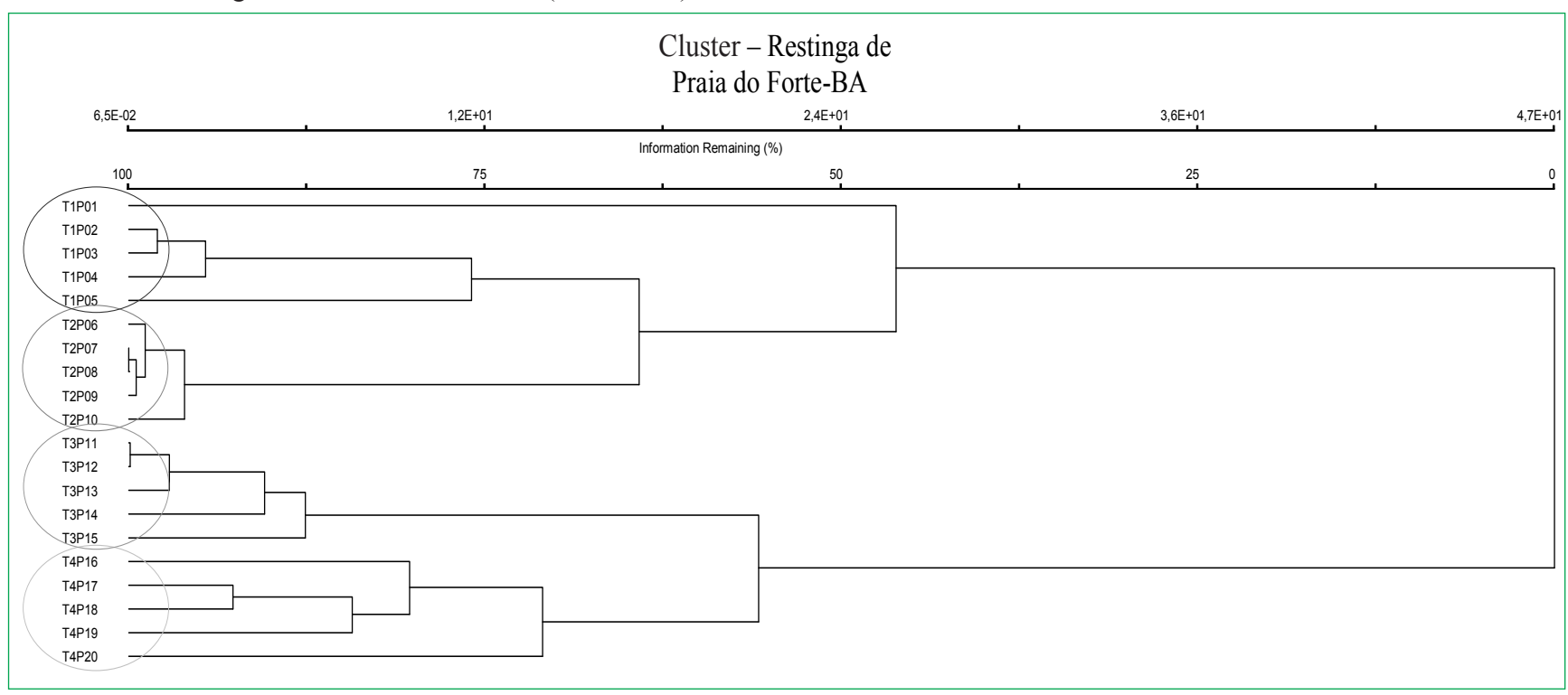


FUNATURA. Plano de manejo da Fazenda Praia do Forte. Relatório Técnico. Brasília: Fundação Pró-Natureza, 1987. 96 p.

HENRIQUES, R. P. B.; ARAÚJO, D. S. D.; HAY, J. D. Descrição e classificação dos tipos de vegetação da restinga de Carapebus, Rio de Janeiro. Revista Brasileira de Botânica, São Paulo, v. 9, p. 173-189, 1986.

IBGE. Flora das restingas do Litoral Norte da Bahia - Costa dos Coqueiros e Salvador. 2004. Disponível em <ftp://geoftp.ibge.gov. $\mathrm{br} /$ documentos/recursosnaturais/levantamento/florarestinga.pdf $>$. Acesso em: 22 fev. 2004.

KREBS, C. J. Ecological methodology. 2. ed. New York: Harper Collins Publishers, 1999. 620 p.

LORENZI, H. Árvores brasileiras: manual de identificação e cultivo de plantas arbóreas nativas do Brasil. v. I. Nova Odessa: Plantarum, 1992a. 352 p.

LORENZI, H. Árvores brasileiras: manual de identificação e cultivo de plantas arbóreas nativas do Brasil. v. II. Nova Odessa: Plantarum, 1992b. 352 p.

LORENZI, H.; SOUZA, H. M.; MEDEIROS-COSTA, J. T.; CERQUEIRA, L. S. C.; VON BEHR, N. Palmeiras no Brasil nativas e exóticas. Editora Plantarum: Nova Odessa, 1996. 303 p.

MARTINS, S. E.; ROSSI, L.; SAMPAIO, P. S. P.; MAGENTA, M. A. G. Caracterização florística de comunidades vegetais de restinga em Bertioga, SP, Brasil. Acta Botanica Brasilica, Feira de Santana, v. 22, n. 1, p. 249-274, 2008.

MENEZES, C. M. Influência da evolução quaternária na vegetação de restinga no Litoral Norte da Bahia. 2007. $94 \mathrm{f}$. Dissertação (Mestrado em Geologia) - Universidade Federal da Bahia, Salvador.

MENEZES, C. M.; AGUIAR, L. G. P. A.; ESPINHEIRA, M. J. C. L.; SILVA, V. Í. S. Florística e fitossociologia do componente arbóreo do município de Conde, Bahia, Brasil. Revista Brasileira de Biociências, Unitau, Taubaté, v. 15, n. 1, p. 44-55, 2009.

MENEZES, C. M.; FARIA, G. A. Diagnóstico da vegetação terrestre da área de influência direta do futuro empreendimento Iberostar, Praia do Forte. Relatório Técnico - Estudo de Impacto Ambiental/Relatório de Impacto ao Meio Ambiente do Futuro Empreendimento Iberostar. Mata de São João, Bahia. 2004. 40 p.

MONTEZUMA, R. C. M. Estrutura da vegetação de uma restinga de Ericaceae no município de Carapebus - RJ. 1997. 102 f. Dissertação (Mestrado em Ecologia) - Universidade Federal do Rio de Janeiro, Rio de Janeiro. 1997.

MULLER-DOMBIS, D.; ELLENBERG, H. Aims and methods of vegetation ecology. New York: Jonh Wiley and Sons, 1974. 574 p.
PEREIRA, O. J.; THOMAZ, L. D.; ARAÚJO, D. S. D. Fitossociologia da vegetação ante dunas da restinga de Setiba/ Guarapari e em Interlagos/Vila Velha, ES. Boletim do Museu de Biologia Mello Leitão, Santa Teresa, v. 1. p. 65-75, 1992.

PINTO, G. C. P.; BAUTISTA, H. P.; FERREIRA, J. D. C. A. A restinga do litoral nordeste do Estado da Bahia. In: LACERDA, L. D.; ARAÚJO, D. S. D.; CERQUEIRA, R.; ROCHA, C. F. D.; ESTEVES, A.; SCARANO, F. R. (Org.). Restingas: origem, estrutura e processos. Niterói: CEUFF, 1984. p. 195-216.

QUEIROZ, E. P. A subfamilia Faboidea (Leguminosae) nas restingas da costa norte do estado da Bahia. 2001. 214 f. Dissertação (Mestrado em Ciências, Área de Botânica) Universidade Federal da Bahia, Salvador. 2001.

RIZZINI, C. T. Tratado de fitogeografia do Brasil. v. 2. 2. ed. São Paulo: HUCITEC EDUSP, 1997. 374 p.

SACRAMENTO, A. C.; ZICKEL, C. S.; DE ALMEIDA JR., E. B. Aspectos Florísticos da Vegetação de Restinga no Litoral de Pernambuco. Revista Árvore, Viçosa, v. 31, n. 6, p. 1121-1130, 2007.

SCHERER, A., MARASCHIN-SILVA, F. E \& BAPTISTA, L. R. DE MOURA. Florística e estrutura do componente arbóreo de matas de Restinga arenosa no Parque Estadual de Itapuã, RS, Brasil. Acta Botanica Brasilica v.19 n. 4, p. 717-726. 2005.

SILVA, S. M. As formações vegetais na planície litorânea da Ilha do Mel, Paraná, Brasil: composição florística e principais características estruturais. Tese (Doutorado em Biologia Vegetal) Universidade Estadual de Campinas, Campinas. 1998.

SONEHARA, J. S. Aspectos florísticos e fitossociológicos de um trecho de vegetação de restinga no Parque Estadual do Rio da Onça - Matinhos, PR. 2005. 77 f. Dissertação (Mestrado em Botânica) - Universidade Federal do Paraná, Curitiba. 2005.

SOUZA, V. C.; LORENZI, H. Botânica sistemática: guia ilustrado para identificação das famílias de Angiospermas da flora brasileira, baseado em APG II. Instituto Plantarum, Nova Odessa, SP, 2005. $640 \mathrm{p}$.

ZALUAR, H. L. T. Espécies focais e a formação de moitas na restinga aberta de Clusia, Carapebus, RJ. Rio de Janeiro. 1997. 105 f. Dissertação (Mestrado em Ecologia) - Universidade Federal do Rio de Janeiro, Rio de Janeiro. 1997.

ZALUAR, H. T. L.; SCARANO, F. R. Facilitação em restingas de moitas: um século de buscas por espécies focais. In: ESTEVES, F. A.; LACERDA, L. D. (Ed.). Ecologia de restingas e lagoas costeiras. Rio de Janeiro: NUPEM/UFRJ, 2000. p. 3-23. 